\title{
Upgrading a Hybrid Computing Algorithm
}

\author{
Researchers outline a protocol for performing a popular \\ quantum-classical machine-learning algorithm with a so-called \\ measurement-based quantum computer, which could allow for more \\ resource-efficient calculations.
}

By Katherine Wright

$\mathrm{M}$ uch like toddlers turning two, researchers working on quantum computers have reached that awkward "in-between" phase: They are beginning to understand the full potential of what they can do, but achieving that potential is - tantalizingly-just out of reach. Quantum computers have issues executing arbitrarily long computations because of hardware limitations, and poorly executed algorithms can easily deliver unexpected-and often incorrect-outcomes.

One route that researchers are taking to guide quantum computers through this early phase is to run their algorithms on

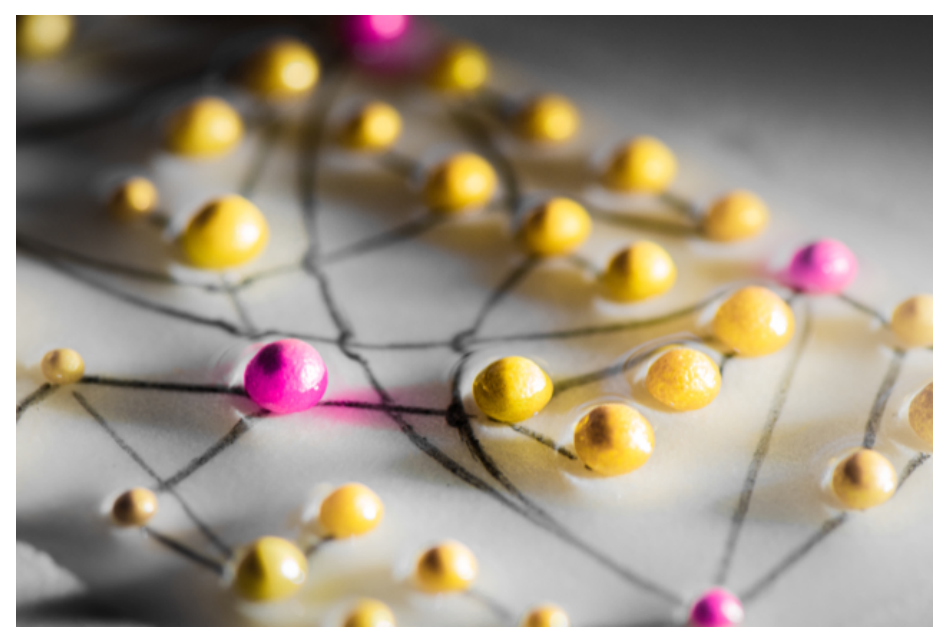

A graph-based approach to quantum computing could make better use of qubit resources than typical circuit-based approaches.

Credit: L. Dellantonio/University of Waterloo hybrid devices that merge a quantum toddler with its more mature sibling-a classical computer. Using machine-learning techniques, researchers can pair classical and quantum processors in feedback loops to solve hard optimization problems. Now, Ryan Ferguson and Luca Dellantonio at the University of Waterloo, Canada, and colleagues outline how to perform one popular quantum machine-learning algorithm on a hybrid system where the quantum processor is "measurement based" [1]. Dellantonio says that their proposal could allow better use of photonic platforms in hybrid computers.

The type of machine-learning algorithm that the team studies is called a variational quantum eigensolver, or the catchy "VQE" for short. VQE algorithms calculate the ground-state energy of a molecule and are specifically designed for hybrid computers, delegating tasks between the quantum and classical processors. Typically, the quantum processor takes a first "guess" at the ground-state wave function of the molecule, encoding that guess into its qubits and then estimating that wave function's energy by performing measurements on the qubits. The classical processor then adjusts the parameters of the guessed wave function to find options with lower energies.

Researchers have demonstrated VQE algorithms for hybrid systems that use "circuit-based" quantum processors, which-like classical processors-perform calculations with gates. The protocol that Ferguson, Dellantonio, and their colleagues outline instead leverages a measurement-based quantum processor, one that works without gates. 
Measurement-based quantum computers-first imagined 20 years ago-perform calculations by creating an entangled quantum state on which a series of measurements are conducted on individual qubits in the state. Hybrid computers that use measurement-based quantum processors are attractive as they potentially allow the quantum part to perform more complex calculations with far fewer qubits than circuit-based versions, Dellantonio says. He and his colleagues show that for a calculation that might require hundreds of thousands of qubits on a current circuit-based system, their measurement-based approach might only need 20 or so qubits.

In the team's protocol, the quantum calculations are done with a so-called graph state, which is a multiqubit state that is typically depicted by a network diagram with vertices and edges. The vertices represent individual qubits and the edges link qubits that interact. First, an "ansatz" graph state is created, which represents the initial guess for the ground state of the system of interest. The initial graph state is expanded by adding qubits and then measuring them. The results of those measurements are fed into the classical computer. A new ansatz graph state is then created, and the process is repeated.

The measurements from the repeated cycles effectively give a series of snapshots of the state's energy for different input parameters. These snapshots are then used by the classical computer to map out the landscape of the energy and pinpoint the minimum. The process is similar to that used for circuit-based quantum processors, Dellantonio says, but the measurement-based method allows more freedom in configuring the qubits that simulate the ground state.

For now, the new protocol is purely theoretical. Dellantonio says that the group is scoping out the possibility of testing its method-with some modifications-on a circuit-based quantum computer available from industry. He also notes that photonic systems exist that could be used to generate graph states and perform the measurement-based protocol.

The new work marks a fresh approach to VQE, says Dan Browne, a quantum physicist at University College London. The team's measurement-based model potentially provides a better way to implement VQE, he says. "I expect that the research groups and companies developing photonic quantum computing platforms will be keen to test this idea on their devices."

Katherine Wright is the Deputy Editor of Physics.

\section{REFERENCES}

1. R. R. Ferguson et al., "Measurement-based variational quantum eigensolver," Phys. Rev. Lett. 126, 220501 (2021). 